\title{
Analysis of two-dimensional and three-dimensional wakes of long circular cylinders
}

\author{
Bernat Font Garcia \\ Aerodynamics and Flight Mechanics \\ University of Southampton \\ Email: b.fontgarcia@soton.ac.uk
}

\author{
Gabriel D. Weymouth \\ Fluid Structure Interactions \\ University of Southampton \\ Email: g.d.weymouth@ soton.ac.uk
}

\author{
Owen R. Tutty \\ Aerodynamics and Flight Mechanics \\ University of Southampton \\ Email: o.r.tutty@ soton.ac.uk
}

\begin{abstract}
The wake behind a bluff body constitutes an intrinsically three-dimensional flow and it is known that twodimensional simulations yield to an unphysical prediction of the body forces because of the nature of the two-dimensional NavierStokes equations. However, three-dimensional simulations are too computationally expensive for cases such as marine risers, which have very large aspect ratios and are exposed to a high Reynolds number flow. A quantitative and qualitative study has been performed to investigate the fundamental differences on the wake of two-dimensional and three-dimensional fixed spanwise periodic cylinders for a Reynolds number of $10^{4}$. A very fine unifrom grid (503M points) has been used for the near and mid wake range, and it is shown that the wake presents very different vortical structures when the spanwise dimensionality is omitted. In this case, forces such as lift and drag are overpredicted. The kinetic energy spectra of the flow is also investigated to further discuss the physics inherent of each case together and it is found that the contribution of the spanwise velocity on the large wavenumbers is significantly smaller than the other velocity components.
\end{abstract}

Keywords-marine risers; computational fluid dynamics; twodimensional turbulence; strip theory

\section{INTRODUCTION}

Marine risers are long and flexible cylindrical structures that extract oil and gas from the subsea oil fields to the surface. Under ocean currents, they are exposed to VortexInduced Vibrations (VIV), a phenomenon arising for long, thin and flexible body shapes. Alternate shedding of vortices from the upper and the lower sides of the circular section of the riser (known as von-Kármán vortex street) creates an unsteady asymmetrical load pattern that stimulates a vibrational state of the riser. This endangers the structure to severe fatigue reducing its lifespan significantly. Besides the VIV phenomenon, Wake-Induced Vibrations (WIV) also come into the picture for structures composed by multiple risers, adding notable complexity to the problem. The offshore industry currently relies on experimental data to derive semi-empirical structural and hydrodynamic models to estimate fatigue damage and the structural failure. Experimental databases need to be repopulated every time new conditions are considered for a novel design. Thus, reliable numerical predictions are of interest in this field.

Risers operate at high Reynolds numbers $\left(10^{4}-10^{6}\right)$ and have a large extent $\left(\sim 10^{3} \mathrm{~m}\right)$ making fully resolved three- dimensional simulations unaffordable. Additionally, the FluidStructure Interaction (FSI) has to be taken into account by iteratively solving the fluid and the solid equations, which further increases the computational requirements. Consequently, academic numerical studies tend to use shorter spans or lower Reynolds regimes. For example, C. Evangelinos and G. Karniadakis [1] investigated an infinitely long (spanwise periodic), rigid and vibrating cylinder for $R e=1000$. $\mathrm{R}$. Bourguet, G. Karniadakis, and M. Triantafyllou [2] studied a flexible cylinder with aspect ratio $(L / D$, where $L$ is the axial extent and $D$ the diameter of the cylinder respectively) of 200 allowed to vibrate in the in-line and cross-flow directions at the $100<R e<1100$ regime by means of a Direct Numerical Simulation (DNS). D. Lucor, L. Ilemas, and G. Karniadakis [3] studied the effect of the oncoming flow profile on a flexible cylinder in the transverse direction for an aspect ratio of 500 - 1000 and $R e=1000$ using DNS as well.

Simulations involving high Reynolds numbers and large aspect ratios cylinders are (currently) only possible by either using coarse grids, specially in the spanwise direction, or by using a strip theory method. The former option was selected by K. Huang, H.-C. Chen and C.-R. Chen [4] to investigate risers at the order of $R e=10^{4}$ and $L / D=1000$. Grids up to $1.5 \mathrm{M}$ cells with coarse spanwise spacing (highest $L / D$ corresponded to $h_{z}=33 D$, where $h_{z}$ is the spanwise spacing) were used. Also in this fashion, S. Holmes, O.H. Oakley and Y. Constantinides [5] studied a similar case and concluded that the coarse resolution of the grid yields discrepancies on the prediction of the forces on the cylinder. The use of such a coarse spanwise spacing is usually justified by stating that the cross-flow vibrations of the risers extends the spanwise correlation length significantly and hence lower resolutions are required. Even though this has been proven for the spanwise length of the excited structural vibration mode (which can go to the order of the cylinder extent), the largest spanwise correlation length of the turbulent wake of the flow is below the order of the diameter. Since only very large scale structures in the spanwise direction are captured and these are much bigger than the actual largest spanwise scale of the turbulent wake, the underresolution of the wake in the axial direction leads to unphysical effects [5].

On the other hand, there have also been numerous studies on the strip theory method. The general approach is to 
place two-dimensional planes along the span of the cylinder which are coupled with a structural dynamic model and/or hydrodynamically using an inviscid unsteady vortex lattice [6], [7]. Because flow past a long cylinder is characterized by a certain spanwise periodicity (see [8]), tackling the problem from a two-dimensional point of view becomes reasonable since two-dimensional planes can be located at grounded positions along the span. Also, because only two-dimensional simulations are required, the computational cost is much cheaper than the three-dimensional approach. This method has been investigated by R.H.J. Willden and J.M.R. Graham [6], [7] and applied to risers up to $L / D=1544 R e=2.84 \cdot 10^{5}$. A Large Eddy Simulation (LES) was employed to only resolve the large scales of turbulence while the subgrid scales were modelled by the classic Smagorinsky gradient diffusion model. C.T. Yamamoto et al. [9], J.R. Meneghini et al. [10] and L. Sun et. al. [11] also used strip theory together with a discrete vortex method (DVM).

The main shortcoming of strip theory methods is that the flow is not allowed to develop on the axial direction and, because of this, flow physics such as turbulence behave differently to three-dimensional flows. This induces forces on the cylinder which do not match the three-dimensional case. Therefore, an accurate prediction of VIV and the structural failure of the cylinder is very challenging. From a physical point of view, this is related to the vortex-stretching mechanism, which causes the turbulent structures to break down into smaller and smaller scales in three-dimensions but is missing in the two-dimensional formulation of the Navier-Stokes equations. Because of this, two-dimensional simulations force vortices to only experience merging and thinning events which cause them to grow in size instead of breaking down into smaller scales. Altogether, this creates vortical structures of larger scale and larger circulation (intensity) than in the threedimensional case causing higher forces and vibrations on the structure.

To attempt to include these effects, Y. Bao et al. [12] employed a "thick" strip theory method. Instead of using twodimensional planes, these had a certain thickness allowing the flow to develop in the axial dimension for short spans. As in the classic strip theory method, the thick planes where placed along the extent of the cylinder. This, however, makes the computation more expensive again since the thickness of the strip must be enough to resolve the spanwise correlation length of the flow.

To be able to model three-dimensional wakes effectively, we must first understand the differences between the twodimensional and the three-dimensional flow. In this work, we analyse the difference between the two-dimensional and the three-dimensional flow around a fixed spanwise periodic circular cylinder at $R e=10^{4}$ using a very fine grid for the near and mid wake regions. The next section will review the equations solved and the numerical methods employed, as well as the computational details of the simulations. In Section III, results regarding the wake of the cylinders will be analysed together with the body forces and the turbulent kinetic energy spectra of different simulations. Finally, a discussion on how to address the two-dimensionality of the flow for the strip model will follow and conclusions will be established on the last section.

\section{NUMERICAL METHODS}

\section{A. Solver}

The continuity equation (1) and the incompressible NavierStokes equations (2) have been numerically solved together with a Pressure-Poisson Equation (PPE) to couple the fluid pressure and velocity,

$$
\begin{gathered}
\frac{\partial u_{i}}{\partial x_{i}}=0 \\
\frac{\partial u_{i}}{\partial t}+u_{j} \frac{\partial u_{i}}{\partial x_{j}}=-\frac{\partial p}{\partial x_{i}}+\frac{1}{R e} \frac{\partial^{2} u_{i}}{\partial x_{j}^{2}} \quad i, j=1,2,3,
\end{gathered}
$$

where $u, v, w$, are the velocity components and $p$ is the pressure field. The Reynolds number is then defined as

$$
R e=\frac{U D}{\nu} .
$$

Immersed Boundary (IB) methods have been extensively used (see [15] and [16] for an extended review) to treat complex and moving geometries as they do not require a fitting grid on the body. The body force on the fluid is usually taken into account by an extra term on the Navier-Stokes equations. The Boundary Data Immersion Method (BDIM) from G.D. Weymouth and D.K.P. Yue [13] (recently extended to compressible flow in [14]) is an IB method which has been used to take into account the solid boundary condition of the cylinder in a non-conforming rectilinear grid. Instead of using an extra term in the governing equations, the BDIM takes into account an interface between the solid and the fluid parts of the domain mapping the governing equations of both parts on it to ensure a continuous transition between the mediums. In this way, metaequations are derived and sharp discontinuities of the solid boundaries which can trigger numerical issues are avoided.

An in-house code which has the BDIM implemented and is fully parallelised has been used to run the simulations. It uses a rectilinear staggered grid, it is second-order accurate in space (QUICK scheme) and second-order accurate in time (predictor-corrector scheme).

\section{B. Computational details}

A rectangular domain of $(35 \times 20 \times 10) D$ in the $x, y, z$ Cartesian coordinate system has been considered for the threedimensional case, omitting the spanwise direction for the twodimensional simulation (Figure 1). A large Cartesian fine grid has been constructed around the cylinder and its wake with uniform spacing to resolve most of the turbulent scales and for post-processing purposes as well. The inner grid starts $4 D$ upstream and ends $20 D$ downstream of the cylinder with $4 D$ above and below the cylinder axis. It is extended all the way across the span of the cylinder. With a resolution of $D=64$ grid points, the inner grid has a size of $503 \mathrm{M}$ points approximately. This gives a non-dimensionalised spacing of 


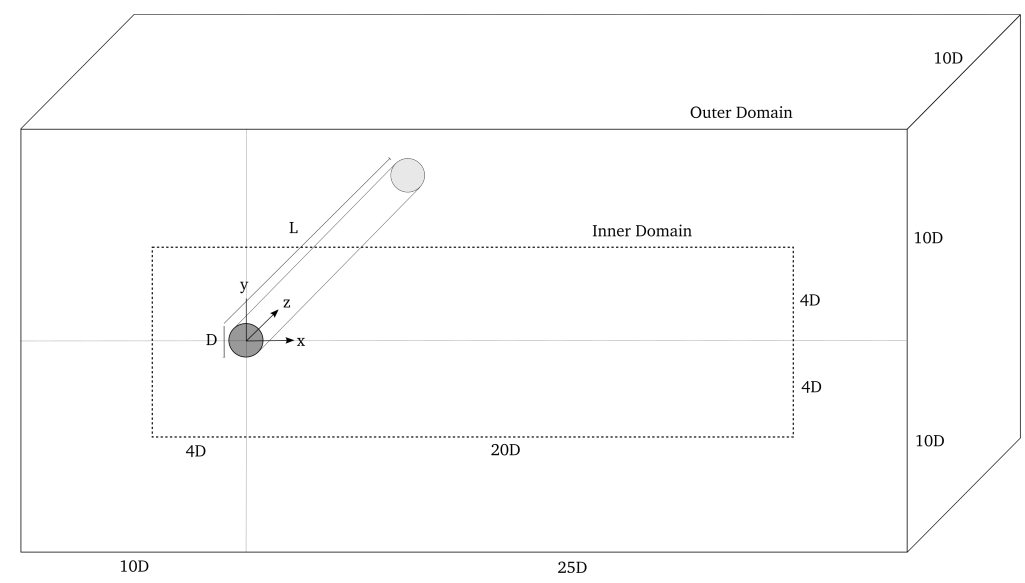

Fig. 1. Computational domain.

$h / D=0.016$ for the entire near and mid wake region. The grid is gradually stretched to the boundaries of the computational domain, where less resolution is required. The total grid size is $734 \mathrm{M}$ grid points.

The smallest scale of turbulence (the Kolmogorov scale) for our case $\left(R e=10^{4}, h=D / 64\right)$ is approximately $\eta / D=$ 0.001 . This means that we are resolving scales of turbulence 16 times bigger than the Kolmogorov scale. The sub-grid scales (SGS) are implicitly modelled using the numerical diffusion inherent in the numerical schemes (Implicit LES).

A uniform velocity profile is considered as the inlet boundary condition. A natural convection condition is considered for the outlet boundary. The upper and lower boundaries are non-reflective. Periodicity is considered for the $z$ direction and the cylinder is fixed. The three-dimensional case has been simulated on the Iridis 4 cluster using 512 cores and a total of 21 cycles of the fully developed time-periodic wake have been computed.

\section{RESULTS}

\section{A. Wake flow}

The visualization of the flow for the two- and the threedimensional cases is reviewed in this section. The wake in the inner domain is showed for the two-dimensional case (Figure 2 ) and the three-dimensional one (Figure 3). For the twodimensional simulation, it can be appreciated that even at a high Reynolds number, the wake still presents strong coherent structures. Vortex-pairing creates couples of counter-rotating vortices which are found along the wake. Since vortex pairs affect each other, once shed they experience induced velocities from other pairs which make them reach positions far away from the centreline of the wake. Also, vortex-merging and vortex-thinning mechanisms [17] allow them to grow in size and strength as they cannot stretch out of plane.

The three-dimensional wake displays a much more wellmixed behaviour as coherent vortical structures are now more difficult to generate. Since the vortex-stretching term is present in the three-dimensional Navier-Stokes equations, turbulent

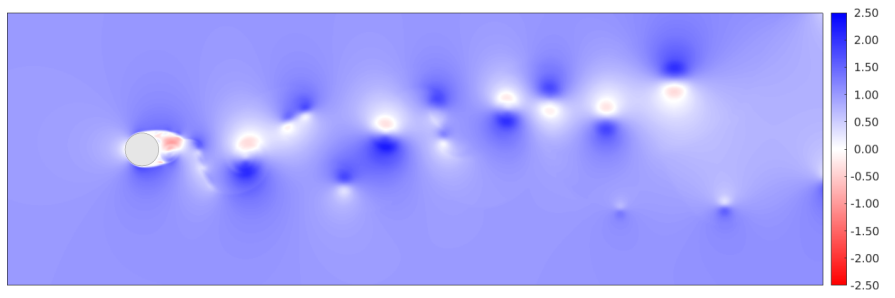

(a) Streamwise velocity $u$.

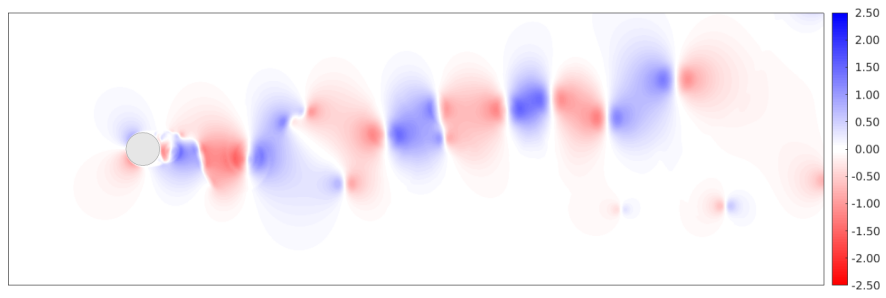

(b) Crosswise velocity $v$.

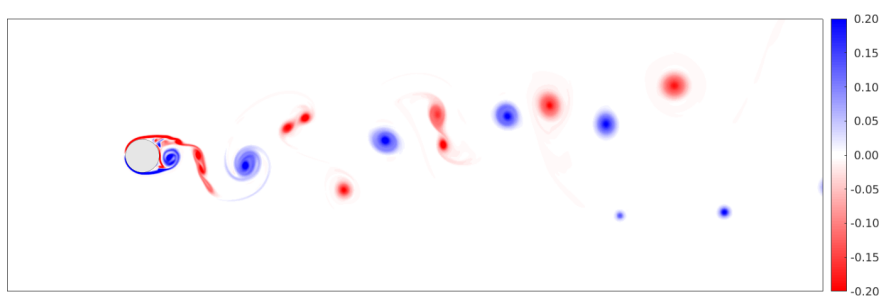

(c) Vorticity $\omega$.

Fig. 2. Snapshot of the two-dimensional case.

eddies stretch on their normal direction reducing their size until they are finally dissipated.

The three-dimensionality of the wake can be observed in Figure 4, where multiple sections along the $z$ direction are shown. The shedding along the span of the cylinder is not totally synchronized. This is a characteristic feature of long fixed cylinders. Also, in order to get a clearer comparison of the wake close to the body, the spanwise vorticity contour for both cases is shown in Figure 5. Besides the smaller vortical structures on the three-dimensional case, it is important to 
note that the roll-up of the shed vortices happens closer to the body on the two-dimensional simulation. This behaviour is well-known (as reported in [18]) and it is one of the main phenomenons which causes the forces on the cylinder to be higher in two-dimensions. In essence, the fluctuating lift force increases as vortices are more intense and they are shed closer to the body than in the three-dimensional case.

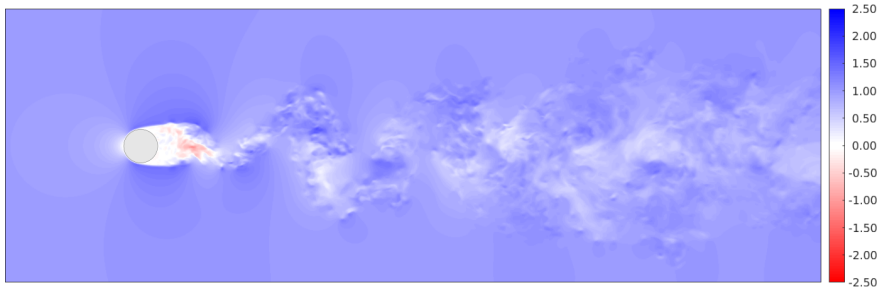

(a) Streamwise velocity $u$.

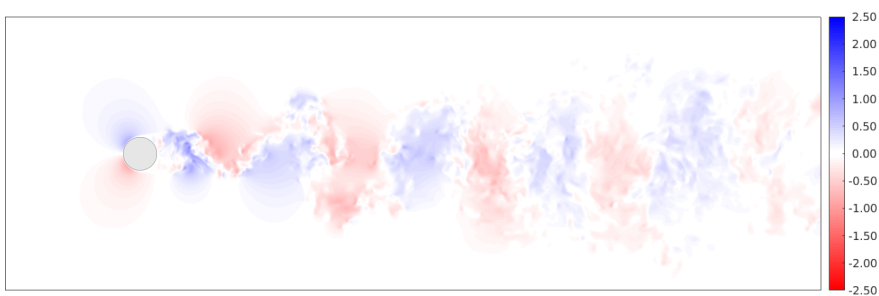

(b) Crosswise velocity $v$.

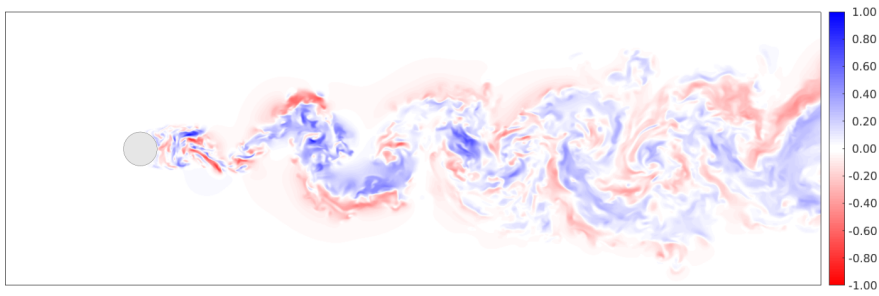

(c) Spanwise velocity $w$.

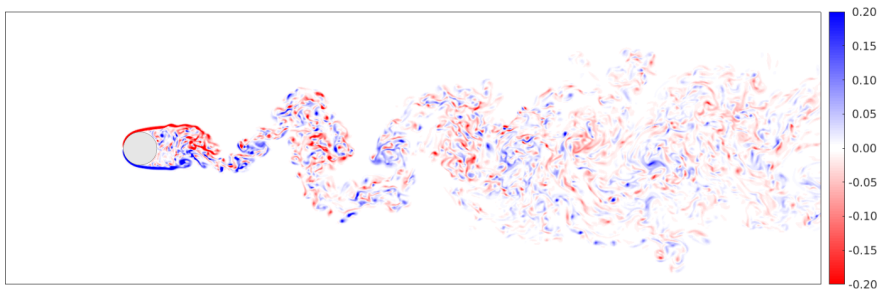

(d) Spanwise vorticity $\omega_{z}$.

Fig. 3. Snapshot of the three-dimensional case. $z / L=0.5$ slice.

In order to asses the validity of simulations using a coarse spanwise resolution, a case with a spanwise grid spacing of $h_{z} / D=0.625$ (only 16 grid points on the span direction) has been simulated. Four slices at different span locations are displayed in Figure 6. The wake does not behave as in the fine three-dimensional case, instead much more coherent vortical structures are found. Even though three-dimensionality can still be observed, the wake resembles a two-dimensional one. As we will show, this also yields to discrepancies on the integrated forces on the body.

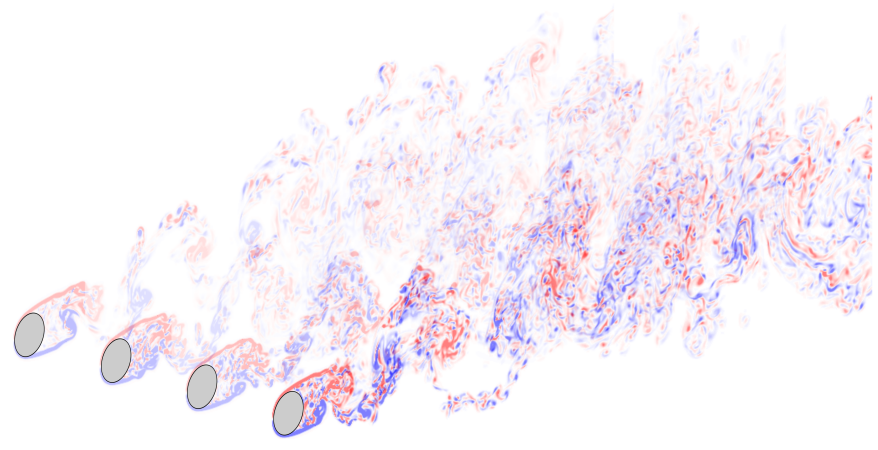

Fig. 4. Slices of spanwise vorticity at $z / L=(0,0.25,0.5,0.75)$.

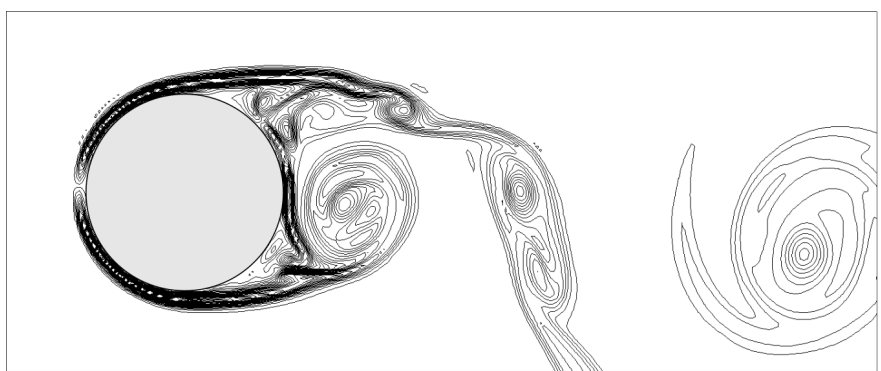

(a)

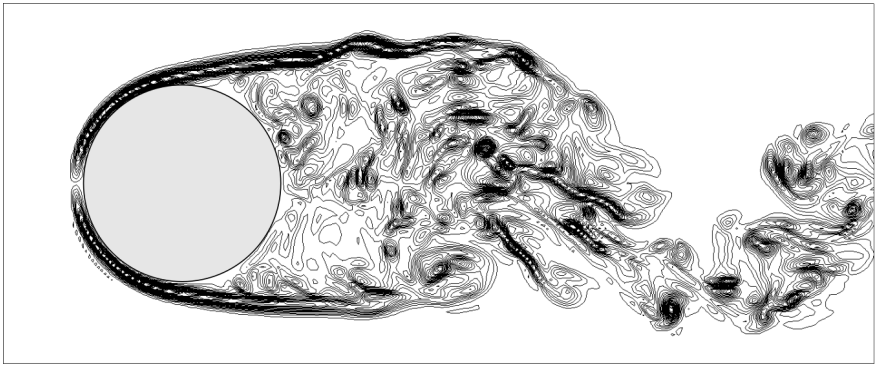

(b)

Fig. 5. Vorticity contour around the cylinder for the (a) two- and (b) threedimensional case.

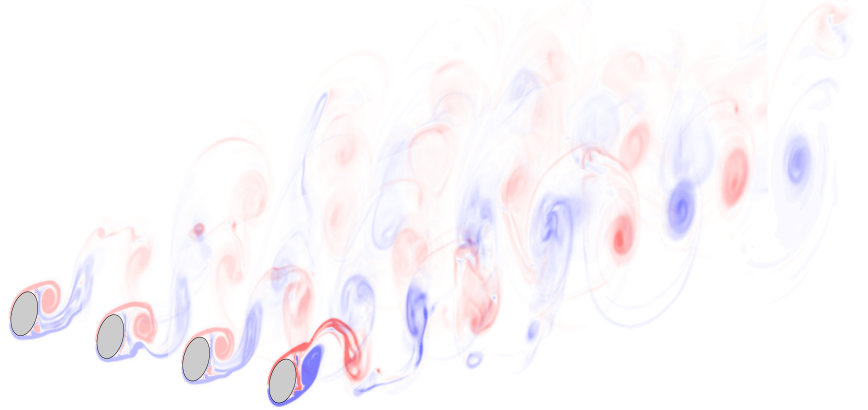

Fig. 6. Slices of spanwise vorticity at $z / L=(0,0.25,0.5,0.75)$ of the coarse $3 \mathrm{D}$ simulation.

\section{B. Integrated forces}

The time evolution of the lift coefficient $C_{L}$ and the drag coefficient $C_{D}$ has been analysed for the different cases and 
these have been computed as

$$
\begin{aligned}
C_{L} & =\frac{F_{y}}{\rho U^{2} D L / 2}, \\
C_{D} & =\frac{F_{x}}{\rho U^{2} D L / 2},
\end{aligned}
$$

where $F_{y}$ and $F_{x}$ are the vertical and horizontal forces on the cylinder respectively. Note that these are forces per unit of span $(L)$, so we can compare them to the two-dimensional forces. Also the shedding frequency $f_{s}$ is discussed in terms of Strouhal number $S t$, which is defined as

$$
S t=\frac{f_{s} D}{U} \text {. }
$$

The shedding frequency has been computed using a Fast Fourier Transform (FFT) of the temporal lift signal allowing us to obtain the dominant frequency.

Figure 7 displays the evolution of both coefficients during the 21 periods of fully developed wake simulated for the three-dimensional case and 24 for the two-dimensional one. The r.m.s. values for each case are summarized in Table I together with the Strouhal number. It can be observed that the fluctuations of the lift coefficient have much larger amplitude for the two-dimensional case than for the three-dimensional ones. In fact, the r.m.s value is more than 5 times higher than the fine three-dimensional case. As explained, this is due to the fact that coherent vortical structures are created in the two-dimensional simulation and these contain higher levels of vorticity. Also, the roll-up of vortices happens closer to the body, hence the shedding of these structures induces higher forces on the cylinder.

TABLE I

SUMMARY OF FORCES, SHEDDING FREQUENCY AND RELATIVE ERROR

\begin{tabular}{|l|c|c|c|c|c|c|}
\hline Case & $\bar{C}_{L}$ & $\varepsilon[\%]$ & $\bar{C}_{D}$ & $\varepsilon[\%]$ & $S t$ & $\varepsilon[\%]$ \\
\hline 2D & 1.09 & 445 & 1.48 & 42 & 0.26 & 24 \\
\hline 3D $h_{z} / D=0.625$ & 0.29 & 45 & 1.31 & 26 & 0.22 & 4 \\
\hline 3D $h_{z} / D=0.016$ & 0.20 & - & 1.04 & - & 0.21 & - \\
\hline
\end{tabular}

Note as well that the drag coefficient presents stronger fluctuations on the two-dimensional case. This is due to the fact that as the vortex shedding arises very close to the body, the near-wake varies its width as vortices are shed from the lower and the upper sides of the cylinder. In contrast, in the fine three-dimensional case the roll-up is found further downstream so the forces do not fluctuate as much and the width of the near-wake is kept almost constant.

For the coarse three-dimensional case, forces present discrepancies compared to the fine case, where the lift and drag coefficients are about $50 \%$ and $30 \%$ overpredicted respectively. Even though the shedding frequency is close to the fine case, this does not mean the solution is physical since the wake visualization and the force coefficients are clearly indicating the contrary.

\section{Kinetic energy spectra}

The kinetic energy spectra has been computed for the different cases in order to identify how is the energy distributed along the length scales of motion (or wavenumbers $k$ ) of the flow. The classic three-dimensional turbulence theory [19] predicts that turbulent kinetic energy flows from large scales (or the scale at which energy is injected) to small scales until the smallest possible scale (the Kolmogorov scale), where the turbulent eddies do not have enough energy to self-sustain and are dissipated because of viscosity. This is the wellknown direct energy cascade of turbulence. And the range of wavenumbers in which turbulent structures receive the same energy from larger scales as they transfer to smaller scales (the inertial subrange) is expected to decay at a $k^{-5 / 3}$ rate.

On the other hand, two-dimensional turbulence experiences a different energy transfer. In fact, energy flows from small to large scales as vortex-stretching (the physical mechanism pointed as responsible for the direct cascade) is not present in two-dimensions. Therefore, two-dimensional turbulence presents an inverse energy cascade. It is also a dual cascade as there is a direct flux of enstrophy cascading towards high wavenumbers at a $k^{-3}$ rate. This is due to the fact that both the total energy $\int E(k) \mathrm{d} k$ and the total enstrophy $\int_{\Omega}|\omega|^{2} \mathrm{~d} \Omega$ are conserved on the non-linear interactions of the inertial subrange and the dual cascade can be observed when the system is fed at an intermediate scale. This scenario is also known as the Kraichnan-Leith-Batchelor (KLB) [20]-[22] theory of two-dimensional turbulence.

The kinetic energy density of the flow is computed in threedimensions as

$$
\bar{E}\left(k_{i}, t\right)=\frac{1}{2} \hat{u}_{i}^{2},
$$

where $\hat{u}_{i}=\hat{u}_{i}\left(k_{i}, t\right)$ are the Fourier coefficients of the velocity components and $k_{i}=\left(k_{x}, k_{y}, k_{z}\right)$ is the wavenumber vector of each spatial direction. Therefore we have computed the FFT of each velocity component which can be written as

$\hat{u}_{i}=\frac{1}{N_{x} N_{y} N_{z}} \sum_{j_{x}=0}^{N_{x}-1} \sum_{j_{y}=0}^{N_{y}-1} \sum_{j_{z}=0}^{N_{z}-1} u_{i}\left(x_{i}, t\right) \mathrm{e}^{-i\left(\frac{j_{x} k_{x}}{N_{x}}+\frac{j_{y} k_{y}}{N_{y}}+\frac{j_{z} k_{z}}{N_{z}}\right)}$,

where $N_{i}=\left(N_{x}, N_{y}, N_{z}\right)$ are the number of points in each direction. The wavenumber vector is defined as

$$
k_{i}=2 \pi \frac{n_{i}}{L_{i}},
$$

where $n_{i}$ is an index going from $\left[-N_{i} / 2, N_{i} / 2\right]$ and $L_{i}$ is the spatial dimension of each direction. The maximum resolved wavenumber is defined by the grid spacing and the minimum by the size of the computational domain. Since only the inner computational domain is used for the FFT, $L_{i}=\left(L_{x}, L_{y}, L_{z}\right)=(24,8,10) D$.

The total energy in function of the wavenumber is obtained calculating the energy density across the wavenumber vector and this can be wirtten as

$$
E(k, t)=\sum_{k-\Delta k / 2<k<k+\Delta k / 2} \bar{E}\left(k_{i}, t\right),
$$




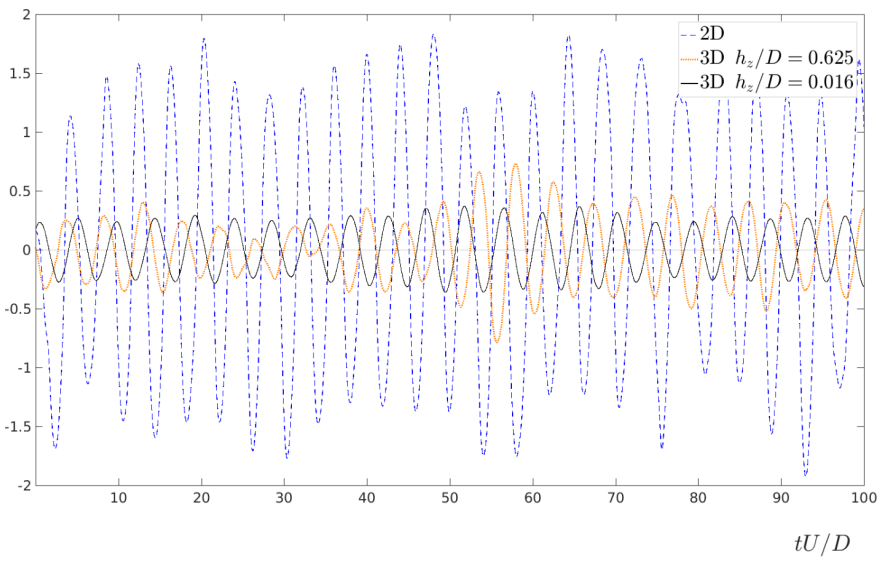

(a) $C_{L}$

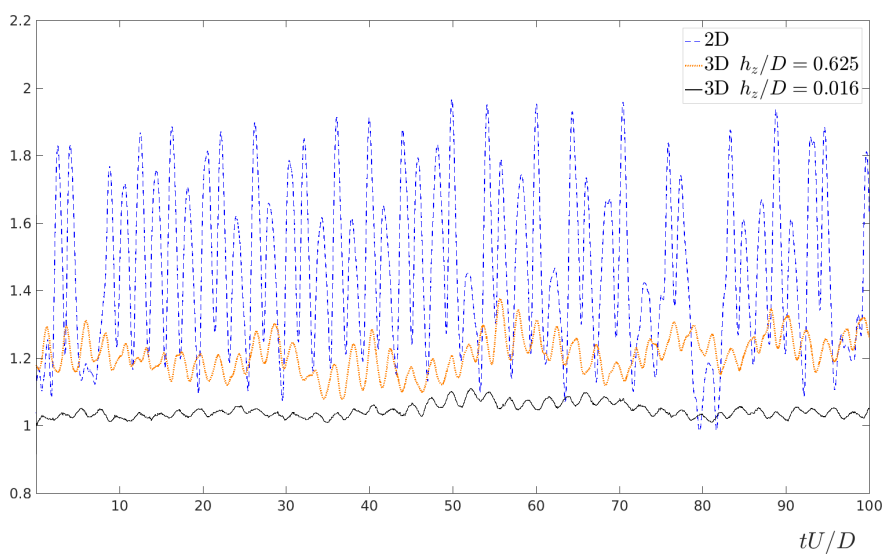

(b) $C_{D}$

Fig. 7. Time evolution of the (a) lift coefficient and (b) drag coefficient as defined in (4) and (5) respectively.

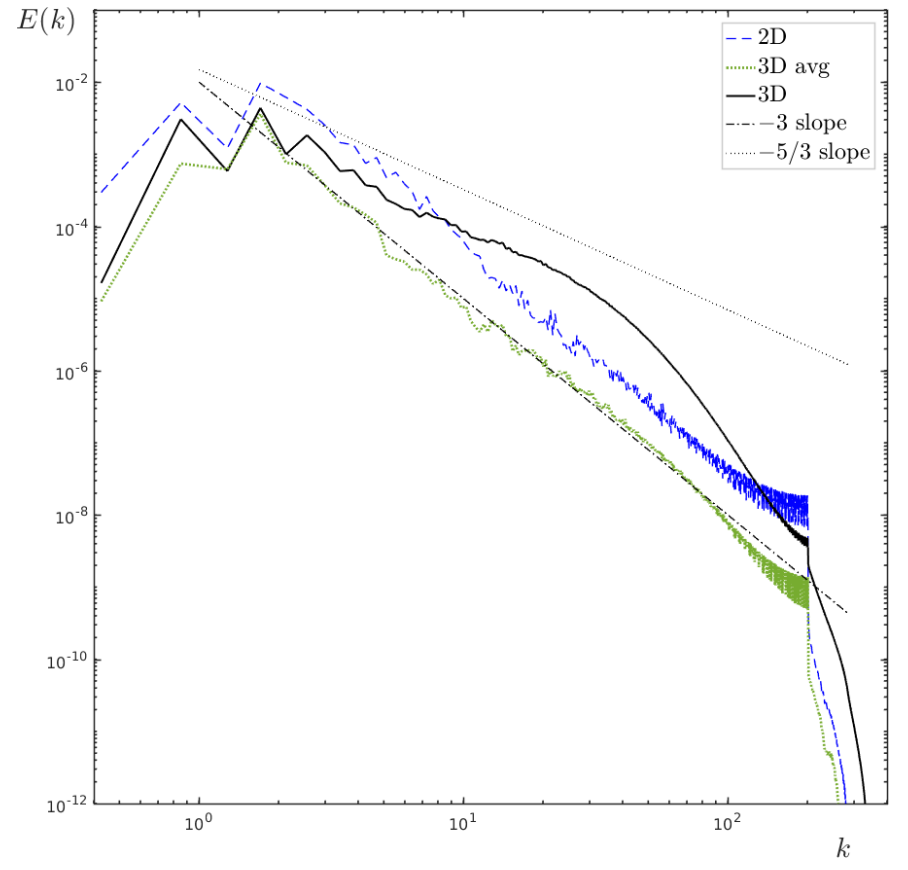

Fig. 8. Turbulent kinetic energy spectra of the two-dimensional, threedimensional and spanwise-averaged three-dimensional case.

where $k=\left|k_{i}\right|$ and $\Delta k$ is the bin size or spectral band used for the wavenumber integration.

It is worth noting that the energy spectra is computed for the total flow (mean and fluctuating quantities). A test was carried in this sense and results were almost identically with or without taking into account the mean flow. Also, as indicated in [23], time-averaged energy spectra of flow past a circular cylinder shows a very similar trend as an snapshot spectra, so this procedure has been omitted as well. Since an FFT expects the input data to be periodic, the periodicity effect of the computational domain was also investigated in [23] by mirroring the FFT domain so that the data set was periodic. However, no significant issues were found hence we have not

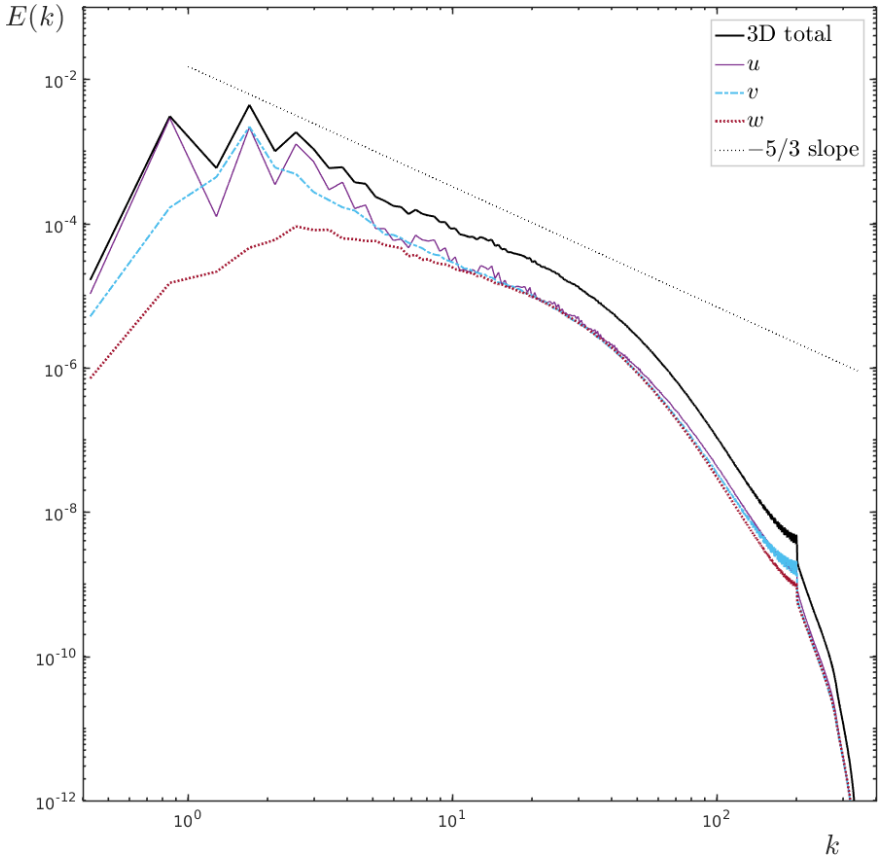

Fig. 9. Turbulent kinetic energy spectra of the three-dimensional case and contribution of each velocity component.

mirrored our data to be periodic.

With this, it can be observed in Figure 8 that the energy cascade of the two-dimensional case behaves the $k^{-3}$ enstrophy decay rate on the inertial subrange. The $k^{-5 / 3}$ slope of the inverse energy cascade is hardly found on the large scales. Similar results were also reported in [23]. Since the energy injection scale is on the order of the diameter and vortical structures of the two-dimensional wake (Figure 2) do not grow much larger than the injection scale, the inverse cascade is difficult to observe. The pile-up of energy on the lowest wavenumber was also captured, however the mean flow data point (lowest wavenumber) is missed from the plot for presentation purposes. It can be also appreciated that there is 


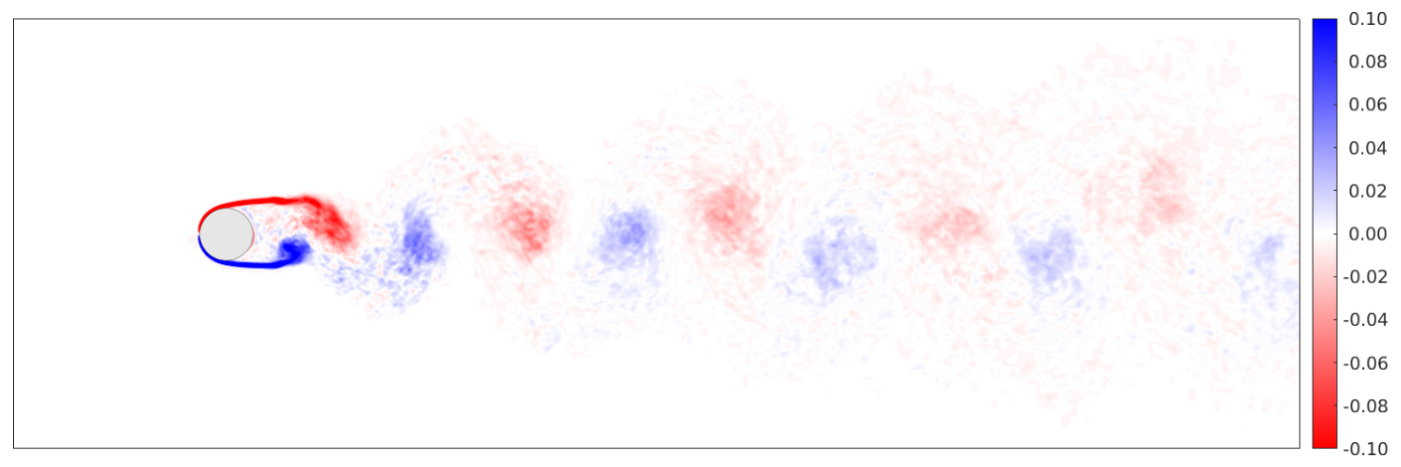

Fig. 10. Spanwise-averaged spanwise vorticity $\left\langle\omega_{z}\right\rangle$ of the three-dimensional case.

a rapid decay for the unresolved wavenumbers.

With respect to the three-dimensional case, this presents a clear direct energy cascade with $k^{-5 / 3}$ on the inertial subrange followed by a steeper slope. Again, there is also a very rapid decay rate found on the unresolved wavenumbers. In this case there is no energy pile-up at low wavenumbers, as expected (all energy is driven to high wavenumbers until it is dissipated). The total energy of the three-dimensional case has been decomposed into its velocity components contribution as shown in Figure 9. It should be noted that the contribution of the spanwise velocity $w$ on the large wavenumbers is significantly smaller than the other velocity components. This demonstrates that coherent structures arising from the cylinder three-dimensionality contain much less energy than the purely two-dimensional structures.

\section{The spanwise-averaged wake}

Driven by the results found in the previous section, the three-dimensional wake has been spatially averaged in the spanwise direction. This allows us to discuss the validity of the two-dimensional simulations by comparing them to spanwiseaveraged flow. The energy spectra of the spanwise-average case can be observed in Figure 8 . A very similar spectra as the two-dimensional case is displayed, so an inertial range of $k^{-3}$ is found as well. The main difference lies on the total energy of the flow, which is not as high as in the two-dimensional case.

The spanwise-average of the spanwise vorticity $\left\langle\omega_{z}\right\rangle$ of the three-dimensional case is shown in Figure 10. It can be appreciated that large (almost) coherent structures are found, contrary to the wake of the pure three-dimensional case (Figure 3). In fact, this could be understood as a 'low Reynolds two-dimensional wake', since the shed vortices are still lying on the centreline of the wake. An important point which can be observed is that the roll-up of vortices still arises further downstream than in two-dimensions and, again, this is one of the key features which dictates the forces on the cylinder. Furthermore, the integrated three-dimensional forces match the spanwise-averaged forces of the cylinder by definition.

\section{CONCLUSIONS}

Two-dimensional and three-dimensional simulations of flow past a fixed spanwise-periodic circular cylinder at $R e=10^{4}$ have been analysed and compared qualitatively and quantitatively. It has been shown that the wake of both cases presents very different behaviours as a consequence of the turbulence nature of the governing equations. It has been shown that, besides the mixed nature of the three-dimensional flow, the roll-up of shed vortices happens closer to the body on the two-dimensional simulation. This yields overpredicted forces compared to the three-dimensional case. It has been also observed that coarse grids on the spanwise direction lead to uncorrelated two-dimensional wakes which, again, overpredict the forces on the cylinder.

The energy spectra of both cases has been investigated as well. The classic direct energy cascade at $k^{-5 / 3}$ could be observed for inertial subrange of the three-dimensional case. The two-dimensional spectra showed a direct enstrophy cascade of $k^{-3}$ and the inverse energy cascade at $k^{-5 / 3}$ was hardly captured. The energy of the three-dimensional case has been decomposed to assess the contribution of each velocity component. The contribution of the spanwise velocity $w$ on the large wavenumbers is significantly smaller than the other velocity components. This proves that coherent structures arising from the cylinder three-dimensionality contain much less energy than the purely two-dimensional structures.

Finally, the three-dimensional wake has been spatiallyaveraged in the spanwise direction and results have been compared to the two-dimensional and three-dimensional wakes. It has been shown that the energy spectra of the spanwiseaverage flow resembles the two-dimensional case. From a visual point of view, the spanwise-averaged wake displays large (almost) coherent structures which look similar to a twodimensional flow. Still, the roll-up of the shed vortices happens far from the body, contrary to the two-dimensional case.

With this, it is clear that pure two-dimensional strip theory methods cannot predict forces around long circular cylinders since the two- and three-dimensional flow behaves differently. Using additional dissipation on two-dimensional simulations can be a way of obtaining lower intensity vortices and, because of this, lower forces. However, it is difficult not to do this 
arbitrarily using a priori models. Instead, we suggest that a spanwise-averaging procedure can be used to tackle the problem. Such procedure can yield to a two-dimensional formulation (hence suited for the strip theory approach) in which the wake close to the body behaves similarly as it does in three-dimensions. Also, by definition, the forces computed from the spanwise-averaged flow match the integrated threedimensional forces. Of course, there are additional physics which would require to be modelled (the spanwise-averaged wake and the three-dimensional one are fairly different on the far wake region), but still this could yield to results closer to the three-dimensional ones.

\section{REFERENCES}

[1] C. Evangelinos and G. Karniadakis, "Dynamics and flow structures in the turbulent wake of rigid and flexible cylinders subject to vortex-induced vibrations," Journal of Fluid Mechanics, vol. 400, pp. 91-124, 1999.

[2] R. Bourguet, G. Karniadakis, and M. Triantafyllou, "Vortex-induced vibrations of a long flexible cylinder in shear flow," Journal of Fluid Mechanics, vol. 667, pp. 342-382, 2011.

[3] D. Lucor, L. Ilemas, and G. Karniadakis, "Vortex dislocations and force distrbution of long flexible cylinders subjected to sheared flows," Journal of Fluids and Structures, vol. 15, pp. 641-650, 2001.

[4] K. Huang, H.-C. Chen, and C.-R. Chen, "Numerical scheme for riser motion calculation during 3-D VIV simulation," Journal of Fluids and Structures, vol. 27, pp. 947-961, 2011.

[5] S. Holmes, O.H. Oakley, and Y. Constantinides, "Simulation of riser VIV using fully three dimensional CFD simulations," in 25th International Conference on Offshore Mechanics and Arctic Engineering, Proceedings of OMAE, 2006

[6] R.H.J. Willden and J.M.R. Graham, "Numerical prediction of VIV on long flexible circular cylinders," Journal of Fluids and Structures, vol. 15, pp. 659-669, 2001.

[7] R.H.J. Willden and J.M.R. Graham, "Multi-modal vortex-induced vibrations of a vertical riser pipe subject to a uniform current profile," European Journal of Mechanics - B/Fluids, vol. 23, pp. 209-218, 2004.

[8] C. Norberg, "Fluctuating lift on a circular cylinder: review and new measurements," Journal of Fluids and Structres, vol. 17, pp. 57-96, 2003.

[9] C.T. Yamamoto, J.R. Meneghini, F. Saltara, R.A. Fregonesi, and J.A. Ferrari Jr., "Numerical simulations of vortex-induced vibration of flexible cylinders," Journal of Fluids and Structures, vol. 19, pp. 467-489, 2004.

[10] J.R. Meneghini, F. Saltara, R.A. Fregonesi, C.T. Yamamoto, E. Casaprima, and J.A. Ferrari Jr., "Numerical simulations of VIV on long flexible cylinders immersed in complex flow fields," European Journal of Mechanics - B/Fluids, vol. 23, pp. 51-63, 2004.

[11] L. Sun, Z. Zong, J. Dong, G.H. Dong, and C.F. Liu, "Stripwise discrete vortex method for VIV analysis of flexible risers," Journal of Fluids and Structures, vol. 35, pp. 21-49, 2012.

[12] Y. Bao, R. Palacios, M. Graham, and S. Sherwin, "Generalized thick strip modelling for vortex-induced vibration of long flexible cylinders," Journal of Computational Physics, vol. 321, pp. 1079-1097, 2016.

[13] G.D. Weymouth and D.K.P. Yue, "Boundary data immersion method for cartesian-grid simulations of fluid-body interaction problems," Journal of Computational Physics, vol. 230, pp. 6233-6247, 2011.

[14] S.C. Schlanderer, G.D. Weymouth, and R.D. Sandberg, "The boundary data immersion method for compressible flows with application to aeroacoustics," Journal of Computational Physics, vol. 333, pp. 440-461, 2017.

[15] C.S. Peskin, "The immersed boundary method," Acta Numerica, pp. 479-517, 2012

[16] R. Mittal and G. Iaccarino, "Immersed boundary methods," Annu. Rev Fluid Mech., vol. 37, pp. 239-261, 2005.

[17] Z. Xiao, M. Wan, S. Chen, and G. Eyink, "Physical mechanism of the inverse energy cascade of two-dimensional turbulence: a numerical investigation," Journal of Fluid Mechanics, vol. 619, pp. 1-44, 2009.

[18] K. Chua, D. Lisoski, A. Leonard, and A. Roshko, "A numerical and experimental investigation of separated flow past an oscillating flat plate," in Nonsteady Fluid Dynamics, pp. 455-464, 1990.

[19] L. Richardson, Weather Prediction by Numerical Process. Cambridge, The University press, 1922.

[20] R. Kraichnan, "Inertial ranges in two-dimensional turbulence," The Physics of Fluids, vol. 10, pp. 1417-1423, 1967.

[21] C.E. Leith, "Diffusion approximation for two-dimensional turbulence," The Physics of Fluids, vol. 11, pp. 671-673, 1968.

[22] G. Batchelor, "Computation of the energy spectrum in homogeneous two-dimensional turbulence," The Physics of Fluids Supplement II, vol. 12, pp. 233-239, 1969.

[23] S.P. Singh and S. Mittal, "Energy spectra of flow past a circular cylinder,” Int. Jl. for Comp. Fluid Dynamics, vol. 18, pp. 671-679, 2004. 